\title{
THE MULTI-RESOLUTION CLEAN
}

\author{
B.P. WAKKER \\ University of Illinois, Department of Astronomy, 1002 W. Green St, \\ Urbana IL61801 \\ U.J. SCHWARZ \\ Kapteyn Laboratory, Postbus 9700AV, Groningen
}

\begin{abstract}
We describe a modification to CLEAN which alleviates some problems for extended sources. This is accomplished by combining the results of a number of conventional CLEAN operations, each done at a different resolution. The algorithm is called "Multi-Resolution Clean" or "MRC". Experiments on model sources have shown that it works well even when the source is so extended that the usual CLEAN becomes impractical. For extended sources, MRC enhances the signal-to-noise ratio, resulting in an easier definition of the area of signal. Moreover, MRC is in principle faster than a standard CLEAN because less $\delta$-functions are needed. This work was published in Astr. Ap., 200, 312 .
\end{abstract}

\section{INTRODUCTION}

CLEAN is optimal for sources which are small compared to the synthesized beam. Problems arise if the source is well-resolved. Then incomplete coverage of the aperture plane (missing baselines, missing or deleted hourangle ranges, unmeasured short-baselines) leads to a complex distribution of artifacts in the map. Further, because CLEAN not only deconvolves the dirty map but also automatically defines the regions with signal, it is unreliable for low signal-to-noise, when $\delta$-functions can be found on noise peaks. For sources of relatively well-known size (like galaxies) one can limit the area where $\delta$-functions are searched-for. This generally greatly improves the reliability of the result. Using a small loop gain can also make the signal-area definition more reliable but the increased number of $\delta$-functions required can lead to a prohibitively long calculation time. Smoothing allows one to improve the signal-to-noise ratio for extended structure, but interesting information is then lost.

A number of other fundamental and practical problems arise for extended sources. First, the extent of the source as measured by the number of independent synthesized beams may not exceed the number of observations (real plus imaginary values). For regular uv-coverage this implies that the area covered by the source should be smaller than the area enclosed within the first grating response. Second, in some cases CLEAN 
can introduce "corrugation", parallel stripes in the map (see e.g. Schwarz 1984). Third, severe problems are caused by missing short-spacings: a) as most of the signal is confined to the inner uv-plane, only a small part of the FT is sampled; b) the zero level in the map will vary and depend on the flux distribution of the source. The latter effect is usually called "the negative bowl". Mapping the source with a single-dish telescope larger than the shortest measured interferometer spacing is the correct way to measure the short-spacing visibilities and remove the bowl (see Schwarz \& Wakker elsewhere in this volume), but this is not always possible.

A number of methods to deal with these problems, using the interferometer data only, have been proposed. Braun \& Walterbos (1986) describe a way to extrapolate the data in the uv-plane. Their method is applicable only for sources that are strongly limited in size and have good uv-coverage. Steer et al. (1984) called an area with intensities above a given level a "component" and convolve that area with the beam. After using an appropriate scale factor, the convolved map is subtracted from the original data and the process is repeated. This leads to a large increase in speed, but for low signal-to-noise it is still difficult to delineate the source. Brinks \& Shane (1984) deconvolved a smoothed dirty map but kept the original resolution by constructing an approximate "correction map" from the difference of the smoothed dirty map and the corresponding smooth clean map. They called this method the "Multi-Resolution Clean". Here we give a more complete and exact description of this method.

\section{CLEANING A SMOOTHED MAP}

A way to lower the number of $\delta$-functions and to clean fainter structure is to smooth the dirty map and beam and then use CLEAN on this map. This produces improvements in three ways:

1) the ratio of source size to beam size becomes better;

2) the signal-to-noise ratio of the smoothed map will be improved;

3 ) by regridding the smoothed map so that the same sampling is kept, smaller maps can be used during CLEANing.

However, one loses the small-scale structure. MRC overcomes this problem by constructing the "difference" map and beam and then using CLEAN on this map too. The process is qualitatively illustrated in Fig 1, for a point source and for an extended source.

The bowl is an extended structure, so it is hardly affected by the smoothing and therefore absent in the difference map. That map contains only the small-scale structure. Sidelobes in the difference map are in general below the noise except for strong peaks, which are usually well separated. The deep sidelobes of the difference dirty beam do not introduce extra problems, whereas the bowl is removed in the smoothed map, where it is easier. Further, the improved signal-to-noise ratio allows to CLEAN the smoothed map much deeper than was possible in the original map; therefore more flux can be recovered. 

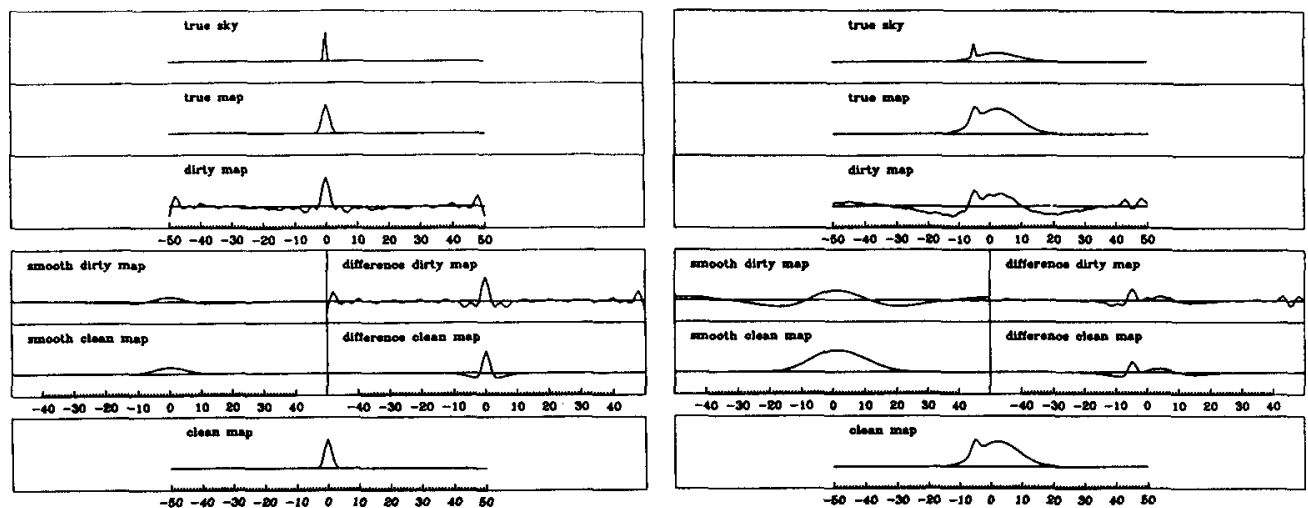

Fig. 1. Profiles showing the steps of MRC for a point source (left) and an extended source plus point source (right). Drawn are the dirty and clean maps for the original, smoothed and difference case. The bowl is evident in the original and smoothed dirty maps of (b). Also note the "sidelobes" in the difference clean map, which make sure that the contribution to the total flux from the difference map is zero.

\section{DESCRIPTION OF MRC}

In order to describe how the clean map at the full resolution is obtained from the smoothed and difference clean map, a number of symbols must be defined. The subscript "s" will stand for the smoothed beam or map $\left(F_{s}=F \star G\right)$ (with $\star$ a convolution), while the subscript " $\mathrm{d}$ " indicates a difference function: $F_{d}=F-F_{q} . \quad G$ is the normalized $\left(\int G(x) d x=1\right)$ smoothing function, whose width is such that the FWHM of the smoothed dirty beam is $f$ times larger than the FWHM of the original dirty beam; $A$ is the dirty beam, with peak value $1 ; D$ is the dirty map; $\delta$ are the clean components; $R$ is the residual after using CLEAN; $B$ is the clean beam with peak value $1 ; C$ is the clean map; $s$ is the scale factor to rescale the smoothed dirty beam back to a peak value $1 ; r$ is the scale factor to rescale the smoothed clean beam back to a peak value 1 . From the $\delta$-functions found by CLEAN one can restore the dirty map by convolving with the dirty beam and adding the residuals. We separate the dirty map into two parts that are treated separately: $D=D_{\mathfrak{s}}+D_{d}$. From this one can derive:

$$
D=\left\{s \delta_{s} \star G+\frac{s}{s-1} \delta_{d} \star(1-G)\right\} \star A+R_{*}+R_{d}
$$

Convolving the $\delta$-functions with a clean beam gives the clean map:

$$
\begin{aligned}
C & =\left\{s \delta_{s} \star G+\frac{s}{s-1} \delta_{d} \star(1-G)\right\} \star B+R_{s}+R_{d} \\
& =\frac{s}{r} \delta_{s} \star B_{s}+\frac{s(r-1)}{r(s-1)} \delta_{d} \star B_{d}+R_{s}+R_{d}
\end{aligned}
$$


MRC requires more parameters than a standard CLEAN: gains and cutoffs used for the smooth and the difference map can be chose independently. A further parameter is the ratio $f$ of the FWHMs of the original and smoothed dirty beams. For relatively small sources, $f=2$ may be best, while for more extended sources a larger $f$ is preferred.

For very extended sources one may use more than one smoothing step. Then the smoothed map would be CLEANed with MRC too. In principle this process can be repeated as many times as wanted, but for practical purposes there is a tradeoff between the optimization of clean parameters and the overhead involved in constructing all the intermediate maps.

\section{DISCUSSION}

The justification usually given for CLEAN is that the sky is essentially empty, with a few small sources scattered around. We extend this assumption by stating: there is a resolution at which the sky appears essentially empty. Schwarz (1978) showed that under this assumption and in the absence of noise CLEAN can in principle recover the unobserved visibilities exactly. This will be true for MRC too. But in the presence of noise it is not possible to obtain the exact result, so (especially for extended sources) MRC can approach the desired result better than CLEAN.

The smoothing of the dirty map can also be done in the uv domain, in the map-making stage, by applying a taper to the uv-data. The difference map is then created by using a taper that is the difference of two taper functions with different widths, such that they cancel each other at the zero spacing. The clean beams should be computed from the same tapers.

In the CLEANing of the difference map short spacings are also recovered. By applying the difference clean beam to the $\delta$-functions they are suppressed when constructing the difference clean map, so that no spurious large-scale structure is introduced.

With MRC one can use a much lower cutoff in the deconvolution process, and therefore it more fully realizes the potential that CLEAN has. Furthermore, the parameters of the several intermediate CLEAN steps can be optimized more than is possible in general. This includes a better signal-to-noise ratio on extended sources, a better source to beam size ratio during CLEANing, smaller maps when CLEANing the smoothed map and a smaller number of $\delta$-functions needed in the difference map.

\section{REFERENCES}

Braun R., Walterbos R.A.M., 1985, Astr. Ap., 143, 307

Brinks E., Shane W.W., 1984, Astr. Ap. Suppl. Ser., 55, 179

Schwarz U.J., 1978, Astr. Ap., 65, 345

Schwarz U.J., 1984, in Indirect Imaging, ed. J.A. Roberts, p255

Steer D.G., Dewdney P.E., Ito, M.R., 1984, Astr. Ap., 137, 159 